\title{
The Process of Compliance with Self-Care among Patients with Hypertension: A Grounded Theory Study
}

\author{
Lubna A. Dwairej*, Muayyad M. Ahmad, Ibtihal A. Alnaimat \\ School of Nursing, University of Jordan, Amman, Jordan \\ Email: ^lubna.dwerij@yahoo.com,mma4jo@yahoo.com,mma4@ju.edu.jo,ibtihalawd@gmail.com
}

How to cite this paper: Dwairej, L.A., Ahmad, M.M. and Alnaimat, I.A. (2020) The Process of Compliance with Self-Care among Patients with Hypertension: A Grounded Theory Study. Open Journal of Nursing, 10, 534-550.

https://doi.org/10.4236/ojn.2020.105037

Received: April 21, 2020

Accepted: May 25, 2020

Published: May 28, 2020

Copyright (c) 2020 by author(s) and Scientific Research Publishing Inc. This work is licensed under the Creative Commons Attribution International License (CC BY 4.0).

http://creativecommons.org/licenses/by/4.0/

\begin{abstract}
Introduction: Controlling hypertension across world continues to be challenging. Managing hypertension is not only concerned with lowering blood pressure by using antihypertensive medications; it also aims to minimize its consequences through adopting self-care practices. Compliance with self-care practices among patients with hypertension is considered a multidimensional phenomenon. The phenomenon of hypertension has been studied quantitatively, however; little qualitative studies were conducted to understand the compliance with self-care among patients with hypertension. Aim: To understand the process that patients with hypertension go through to comply with self-care practices. Methods: This study used a qualitative design that followed constructivist grounded theory approach; purposive sampling was used to recruit participants from cardiac clinics; semi structured, in-depth and face-to-face interview was used as a major method for data collection. Findings: Four participants with hypertension participated in this study; the phenomena of self-care was identified as the central phenomena; the start of the disease was identified as a casual condition; beliefs toward hypertension disease, beliefs toward self-care practices, knowledge and awareness regarding hypertension disease and self-care practices were identified as strategies; experiencing self-care practices was identified as consequence and being patients with hypertension in a social context. Conclusions: The process of compliance with self-care has a path of actions and interactions. The process started from the moment of diagnosis where the patients start to think about self-care. The absence of health care context leads to varying level of compliance with self-care among patients with hypertension. This indicated the need for more effective patient and health care provider relationship, education and awareness campaign.
\end{abstract}




\section{Keywords}

Process of Self-Care, Constructivist Grounded Theory, Hypertension

\section{Introduction}

Controlling hypertension (HTN) across world continues to be challenging. Managing HTN is not only concerned with lowering blood pressure by using antihypertensive medications; it also aims to minimize its consequences through adopting self-care practices [1]. Self-care is defined as "actions directed toward one self or the environment to regulate functioning and well-being" [2]. Compliance with self-care practices among patients with HTN is considered a multidimensional phenomenon [3] since it requires frequent monitoring of blood pressure, regular exercise, and low salt-fat diet, and stress management, weight control, smoking cessation and alcohol avoidance [4]. Self-care was found to be directly associated with blood pressure control [5]. Hypertension self-care was also associated with improvement of patients' quality of life and health outcomes [6] [7].

Hypertension (HTN) is defined as persistent elevation of systolic blood pressure of $140 \mathrm{mmHg}$ or higher and diastolic blood pressure of $90 \mathrm{mmHg}$ or higher [8]. In 2010 the global prevalence of HTN among adult patients was 31.1\% [9]. Additionally, the estimation of the latest worldwide death caused by hypertensive heart diseases is $1.7 \%$ of total death [10]. In the Arab world the estimated crude prevalence for HTN was $29.5 \%$ [11]. Increased blood pressure is considered a serious risk factor for coronary heart diseases, chronic kidney disease, ischemic and hemorrhagic stroke [12].

The phenomenon of HTN has been studied quantitatively; for example many studies conducted have focused on prevalence, awareness, treatment and the control of HTN [9] [13] [14] [15]. Other studies focused on the HTN treatment outcomes and clinical factors associated with HTN control [14] [16] [17] [18]. However, little qualitative studies were conducted to understand the compliance with self-care among patients with HTN.

The aim of this study was to understand the process that patients with HTN go through to comply with self-care practices.

\section{Philosophical perspective}

The focus of grounded theory is on the inductive analysis where the researcher moves from particular to general; that is, researcher generates new hypothesis and theories from many observations [19]. Researchers in the grounded theory do not wait until the end of the data collection to start analysis instead grounded theory requires that the data collection and analysis take place concurrently. Memo writing (memoing) is used to capture researcher's idea about the concepts and ask questions about the data as being collected and analyzed. Data analysis in grounded theory relies on coding and constant comparison [19]. Coding is a 
process of breaking data down into much smaller components and labelling those components. Open coding entails line by line examination of the data to identify concepts and to conceptualize underlying pattern. While in axial coding researcher identifies central phenomena, causal conditions, strategies, context and consequences. Constant comparison entails comparing data with data, event with event, and code with code to explain variation in the data [19]. Through constant comparison, data that represent similar facets of the same concept will be also identified. Theoretical saturation is central to the grounded theory and many other qualitative researches. Theoretical saturation implies that there are no limits to be set for the number of participants, sampling of data continues until saturation is reached. The results of grounded theory are the production of a substantive theory in which a set of concepts are related to one another in a cohesive way. Grounded theory researchers have been divided into three camps; those who follow Glaserian or Classic Grounded Theory, those who follow Straussian Grounded Theory and those who follow constructivist grounded theory [19].

In this study constructivist grounded theory (CGT) was used to study the compliance of Jordanian hypertensive patients with self-care practices [20]. Constructivist grounded theory has it is root in the symbolic interactionism. Unlike other version of grounded theory, Charmaz clearly adopted a constructivist standpoint with relativist ontology and subjective epistemology [21]. In the early $21^{\text {st }}$ century CGT was developed by the sociologist Kathy Charmaz [20]. Charmaz has moved the grounded theory from its original positivist underpinnings to a constructivist one [22].

Consistent with relativist ontology and symbolic interactionism, reality in CGT is socially constructed [21]. That is realty is discovered through interaction process within cultural and social context. It is through the process of interaction with participants and data, researchers influence the research [22]. This interaction process is framed by researcher and participants. Charmaz has advocated for theory that is co-constructed by the researcher and participants. In the current study, the reality was co-constructed during interview through the process of interaction between researchers and patients with HTN.

\section{Literature Review}

Qualitative approach would be appropriate to address the current study phenomena since qualitative approach aims to understand and interpret complex human phenomena [23].

A qualitative study that utilized a constructivist grounded theory framework was conducted to explore how patients with hypertension experience self-management of health care [24]. In their studies Balduino et al. [24] has recruited 28 participants hypertensive patients aged between 18 - 59 years by using theoretical sampling technique. Analysis was conducted by using initial, focused and axial coding. The categories that emerged during the data analysis were organized accord- 
ing to Strauss and Corbin pragmatic model. In Balduino et al. [24] study, five categories have emerged during data analysis process. Hypertensive patients' experience of self-management has emerged as a central category. However, beginning of the disease has emerged as a causal condition. Understanding the disease process and incorporating behavior of self-management were emerged as strategies. Those strategies are actions emerged in response to central category. Experiencing attitudes and actions in the treatment of the disease has emerged as a consequence for the central category. Being treated in the public healthcare system has emerged as contextual condition that influenced the emerged strategy [24].

Another qualitative study has utilized a grounded theory approach aimed to understand how hypertensive patients with associated comorbidities experience self-management and how the beliefs of those patients might influence medication adherence and HTN control [25]. In Fix et al. [25] study, data were analyzed through open and thematic coding; where the NVivo was used to facilitate coding process. Four themes related to HTN management have emerged from analysis. The central theme was interdependence; that is participants have reported that HTN was intertwined with their comorbidities. The second theme was low priority where the participants assign low priority for HTN management compared with other comorbidities. Conflict was also another theme; where the participants have reported that other comorbidities management has conflicted with HTN self-management. The fourth was managing medications multiplicity; where the participants felt confused when taking more than one medication [25].

A recent mixed method study aimed to identify and understand factors influence adherence with antihypertensive medication among patients with hypertension [26]. The results of qualitative study indicated that participants' awareness for increased BP complications was reported as factor that enhanced medication adherence. However, the absence of high BP symptoms and antihypertensive medication side effects were reported as influential factor that interrupted medication adherence [26].

An inductive qualitative study was conducted to understand the perceived benefits and burdens associated with digital interventions designed to enhance self-management in patients with HTN [27]. In this study, seven themes were emerged from data analysis. The first theme was benefit of reassurance from seeing BP reading that is participants felt comfort when their BP readings were under control. The second theme was benefit of motivation for life style changes from seeing BP readings; that is when participants see their BP they are motivated to modify their life style. The third theme was benefit of better health, that is when the health care professionals have an access to participants' BP readings and have noticed a high readings they might promptly change participants medication and this might improve health [27]. The fourth theme was burden of worrying about health; it is the participants' negative feelings when they have 
seen their BP reading uncontrolled and the worry results from medications change. The fifth theme was burden of thinking about making healthy life style changes; it is the blame feeling when not engaging with healthy lifestyle. The sixth theme was burden of uncertainty from self-monitoring; it is the uncertainty about the right actions when the BP is high or low. The last theme was burden of the practicalities of adhering to intervention procedure; that is the burden faced participants to fit self-management with their daily routine [27].

\section{Methodology}

\subsection{Study Design}

This study followed constructivist grounded theory approach [20]. The nature of grounded theory that aims to develop theories that address a social process grounded in real experience would be appropriate to understand compliance with self-care in patients with HTN [28]. Further using CGT approach, helped us to develop a conceptual framework that explain the process of complying to self-care practices in patients with HTN.

\subsection{Sampling Technique}

The participants in this study were patients with HTN who lives in Jordan. At the beginning of the current study the purposive sampling (homogenous) was used to understand how patients with primary HTN comply with self-care practices. Purposive sampling was used to recruit participants who can provide us with rich data of their experience. Since CGT emphasis on the constant comparison and selective method of data obtaining, in the later stages of the current study we used the theoretical sampling to allow for fully developed concepts and theoretical saturation [21]. That is researcher continued to recruit participants until saturation was reached.

In the current study the inclusion criteria were not restricted to allow for the theoretical sampling that follows purposive sampling. However, we included participants with the following criteria:

- Diagnosed with hypertension according to the American Collage for Cardiology/American heart association clinical practice guidelines [29] (systolic blood pressure $>140 \mathrm{mmHg}$ or diastolic $>90 \mathrm{mmHg}$ ).

- Aged more 18 years.

- Have Jordanian nationality.

- Visit cardiac clinics during the data collection period.

Participants with any mental or psychiatric disorder were excluded from the current study.

\subsection{Setting}

The participants were selected from cardiology clinics affiliated with one of the teaching hospital in Jordan in the period from December $1^{\text {st }}, 2019$ to January $1^{\text {st }}$, 2020. 


\subsection{Data Collection}

We used semi structured, in-depth and face-to-face interview that was audiotaped as a major method for data collection. Interview is one of the most common data collection method used in grounded theory research since it provides direct, rich and comprehensive data regarding phenomena of interest [30]. Also, interview will encourage the participants to express the multiple dimensions of phenomenon as they perceive, rather than being directed by the researcher preexisting notion about the phenomena [31]. Unlike unstructured interview, semi structured interview allows us to gain in depth information from participants by using interview guide questions [30]. The interview guide questions will lend focus to the research topic without imposing control or comprising participants' own perspective [30].

Additional information such as non-verbal expression, emotions and environmental situation were collected through field notes. Also, demographic data including participants' age, gender, marital status, educational level, other health care problem and duration for HTN diagnoses were obtained using demographic data sheet before the interview.

\subsection{Data Collection Procedure}

Letters to get permission for conducting the study were sent to the necessary boards, request for permission was sent to ethical committee in the hospital, the permission was requested from the nursing administrators and the in charge personnel in the selected clinics to get an accesses for participants. When these requests were granted the invitation letters were distributed to the target clinics inviting the participants into the study, these letters clarified the aims of the study, participants' rights, confidentiality issues and information how to contact the participants.

The interview consisted of three open-ended questions which were developed by the researchers with further questions and probes that were used as necessary. These questions included:

- Tell me how you came to have HTN?

- As a patient with HTN how do you take care of yourself?

- What are the barriers hinder you from practicing self-care?

Interviews take place in participants' homes and the time of the both interviews ranged from 25 - 30 minutes. The interviews were audio taped after the permission from the participants for analytical purposes and notes were taken during the interview for reflexivity. The confidentiality was assured during the course of the interview. The interviews were transcribed verbatim and were checked for accuracy. Pseudonyms were used to identify participants in all transcripts and reports.

\subsection{Ethical Consideration}

Privacy and confidentiality were preserved all the times, the entire findings were 
depicted confidentially; no identifiable information was recorded or printed. Audio taped interviews were transcribed verbatim; therefore, no names were recorded during the interviewing process. Anonymity is difficult to achieve in such studies which involve interviews. Thus, recorded interviews were deleted when completing the study. To maintain participants' autonomy, we provided the participants with ongoing consent form to sign, the informed consent was insured before starting the interviews and the participants were continuously reminded about their right to withdraw during the interview and not respond to any question without any consequences.

\subsection{Data Analysis and Management}

Our data analysis was analyzed based on constructivist grounded theory approach. Data were analyzed using initial, focused and axial coding. The main strategy of defining properties was constant comparison [20]. The interviews were transcribed into Arabic while listening to the audiotaped. For the accuracy, the transcriptions were read when listening to the record. The interviews were read thoroughly more than one time. After that, initial and focused codes were identified. Later on, data were organized into casual condition, strategies, context and consequence. Data for interviews were read and re-read to check the suitability of identified codes. The codes were done by both researchers until they reached agreement about the final initial, focused and axial codes.

\subsection{Trustworthiness}

Two of Guba and Lincoln criteria for enhancing trustworthiness in qualitative research were followed including; credibility, transferability [32]. To enhance credibility many strategies that are suggested by Shenton [33] were considered. The research adopted a recognized methodology that was successful in previous similar studies in term of the interview questions, data collection procedures as well as data analysis method [33]. Frequent debriefing sessions with the researchers through the data analysis process were also utilized to enhance the credibility. Transferability of data was ensured by providing a detailed contextual data about the study in term of participants, setting the data collection procedure [33].

\section{Findings}

\subsection{Participants' Characteristics}

Four participants with hypertension participated in this study, a 50 years old female participant, 65 years old male participant, 55 years old male participants and a 49 years old female participant. Two of the participants had a diploma degree while the other two participants had bachelor degree. One of the participants has been diagnosed with HTN since one year and the other participants have been diagnosed since more than 5 years. 


\subsection{Analysis}

Based on the findings of the interview the phenomena of self-care was identified as the central phenomena, the start of the disease was identified as a casual condition, beliefs toward HTN disease, beliefs toward self-care practices, knowledge and awareness regarding HTN disease and self-care practices were identified as strategies; experiencing self-care practices was identified as consequence and being patients with HTN in a social context. Figure 1 demonstrates the correlation of categories with the central phenomena; the phenomena of self-care practices to control and manage HTN.

\section{Start of the disease}

Patients with HTN experience many symptoms before being diagnosed. Participants go to the hospital to seek for health care where they receive appropriate diagnosis and treatment before they start practicing self-care process. In this study, this occurs by experiencing HTN symptoms, hospital visits and BP monitoring:

"My hands and legs swelled only and I felt strange heart beat I went to the hospital they monitored my BP, made blood test, kidney function test I remember and diagnosed me with HTN, physician prescribed me a medication, one tablet every day, they did not give me any advices" (P1).

"I felt headache, dizziness, vomiting and went to primary care center, they monitored my BP and was 170/100mmHg. Then I monitor BP for one week and after I visited the internal physician, he prescribed me medication; Amlocard 5 $\mathrm{mg}$, two tablets per day, advised me to avoid salty food and began to care for myself" (P2).

\section{Beliefs toward HTN}

Hypertension was perceived as a common and non-threatening, one participant expressed: "I think that these days all people above 40 years have HTN, what is the matter... may be people in the past did not have HTN why? Because of physical activity" (P1). Other participants viewed HTN from their religious perspective: "as a Muslim... I believe that disease atonement for sins and HTN is from the God Fate and destiny..." (P3).

It was believed that salty and fatty food cause HTN: “...salt lead to increase blood pressure; increased amount of salt in food and butter, that is" (P1). Stress was also believed to cause HTN: "stress impact my BP surly, stress increase BP" (P4).

Hypertension can be managed by medication: "people who comply with antihypertensive medication and comply with avoiding things that cause increased BP can live normally" (P2).

\section{Beliefs toward HTN self-care practices}

Participants with hypertension have a negative feelings regarding antihypertensive medication and believed that antihypertensive medication have a damaging effect on the body: "some kinds of antihypertensive medications were withdrawn from markets because it leads to disease, recent study found that lung 


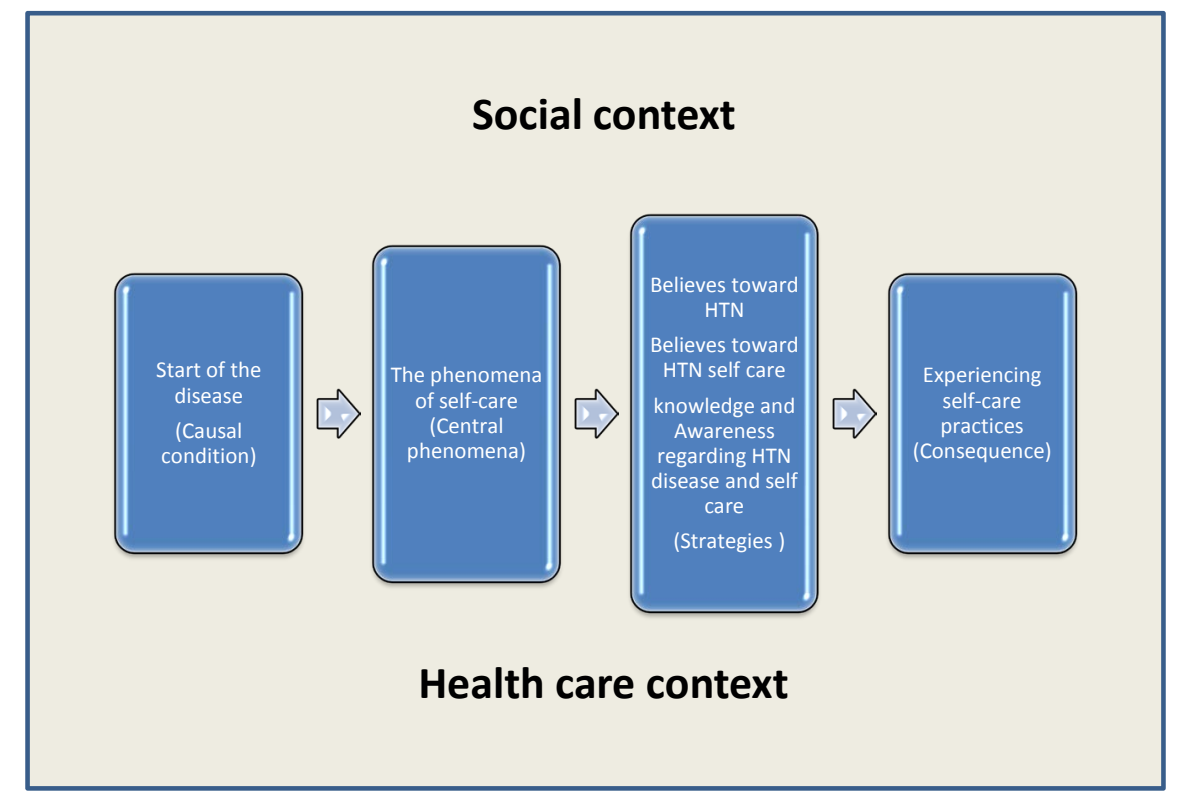

Figure 1. The process of compliance with HTN self-care practices.

cancer are caused by antihypertensive medications... antihypertensive medication have a harm effects..." (P1). Other participant said: "I try to avoid poison come from antihypertensive medications" (P2). However, patients still acknowledge the role of medication: "I think that medication control BP" (P3).

Participants viewed alternative medicine as safe choice and effective in reducing BP: "Roselle, spicy coffee I have read may four or five natural things you can take regularly lead to decrease BP...."; "Herbals are very beneficial for example ginger, cumin, cinnamon and black grain"; "To avoid the chemical of medicine, I like to take things that help me not to take medications... alternatives are made from natural remedies it is better and safer for health" (P4).

One participant viewed no benefit for walking: "antihypertensive medication is enough, why I have to get tired from walking, right?" (P1). While other participant had a different view for physical activity: "physical activity and being active... benefit human body" (P2). One participant belief in the role of stress management: "if I get nervous this thing impacts my health and increases my BP" (P3).

\section{Knowledge and awareness regarding HTN disease and self-care practice}

Most of the time participants get their information from untrusted resources: "I visit spice dealers to benefit from their experience regarding HTN, I confirmed information given by them through internet then I go back to them to further discuss this information..." (P2); "when I open the internets' web pages I find different topics... I become lost in the web pages and do not know what to use and finally do not use anything and return to the medication ...” (P1).

Participant indicated their need for trusted resource of information: "...if there is a clear and right resource one will follow... I will comply with trusted program, program tell me about diet, physical exercise a clear program not done 
by me... I want someone to tell me clearly what to do to control BP... I am ready to use this" (P4)

Participants were aware and searched for the consequences of uncontrolled BP: “...also, I searched what are the complications if BP was not within normal range... I take care for myself to avoid strokes this what threatens one; heart attack" (P1), “...we heard that HTN impact on arteries, and heard about heart attack and stroke, these things when we read or heard about motivate us not to be laze and to take care of our health" (P3).

\section{Experiencing self-care practices}

Participants have focused on the diet aspects and expressed their compliance with HTN diet: "I eat, for example we do not use canned food, and we usually use little salt... I used not to eat butter... I avoid salty food, food with high cholesterol I avoid...” (P1); “...I focus on vegetables and leafy vegetables like Spinach, green beans and parsley" (P2). One participant thinks that she/he is doing excellent with her/his diet: "I am doing enough with diet... the nature of my diet before and after HTN is the same since I used to eat healthy..." (P4).

Participants' comply with antihypertensive medications but it seems that participants were obligated with this compliance: “...I take my medication regularly to avoid HTN complications..." (P1); "despite of medications proven harms I continue to use medication because there is no alternative" (P3); "I wish not to use medication but no solution and no escape... I wish if they find alternative for antihypertensive medication, they might find natural alternative" (P1).

Participants do physical activity in different ways: "I do walking and jogging exercise... I do a lot of activity, I try to be active and do anything in home" (P2). Other participant expressed: "as a women in our homes what physical activity that we have to do, we are doing housekeeping work... we are cooking we are washing dishes..." (P1).

Participants demonstrated varying practices in relation to BP monitoring: "I watch my BP... I used to monitor my BP approximately on a weekly basis" (P2). Other participant stated that: "I do not monitor my BP regularly... I monitor my BP with sphygmomanometer once in two months maybe" (P1).

Participants avoid nervousness: "I try to avoid nervousness... when I felt nervous; I tried to control myself and remembering and ask forgiveness of God usually and remind myself that nervousness impacts my health and increases my BP" (P2). However, there were no specific techniques to control stress: "if one have a stress what could be done? ... No specific techniques for stress management.... One depend on god and do not worry, worries do not solve problem" (P4).

Participants used alternative medicine to control BP: "I find that apple vinegar help in decreasing BP and I have used it for a period... and felt comfort about using apple vinegar.... Fruits; find banana beneficial, Avocado, Orange, Apple these substances have zinc and magnesium help me to control BP within normal range" (P2). 


\section{Being a patient with HTN in a social context}

Participants with HTN gained their knowledge regarding HTN disease and self-care practices from resources within social context: "no one in the hospital give me advices and just we see people around us decreasing salt... I have read on the internet many things to reduce BP... I don't talk with physician or nurses about HTN... we depend on people talks; We rely on what people say regarding things that might increase blood pressure; one of my relative told me about apple vinegar and find it suitable" (P1).

Participant' primary clinics and follow up visits were for the disease diagnosis and for reissuing medications. Health care providers did not provide patients with appropriate health education regarding the disease management: “...we go to the hospital and they diagnosed me with HTN, no one gave me health care advices ..."; "I visit the physician; he/she might change the medication..." (P3).

It seems that participants' personal belief with self-care practices addressed within medical context was strong: "I do not follow a program just the medication" (P1); "I comply with medication and I know that I have to take one tablet daily and use it frequently" (P2). However it seems that participants have little belief and were hesitant regarding self-care given addressed within social context, one participant has expressed the following statements regarding walking and physical exercise: “...they said walking reduce BP, we don't walk... one read a lot of things on internet but finally we do not do anything” (P1) “...so physical activity has a role, we cheating ourselves physical activity has a role... do I make enough regarding physical activity; god know, no, I do not know..." (P1).

\section{Discussion}

The purpose of this study was to understand the process that hypertensive patients go through to comply with self-care practices. This section discusses the study findings. It explores and interprets the casual condition (start of the disease), strategies (beliefs toward HTN disease, beliefs toward self-care practices, knowledge and awareness regarding HTN disease and self-care practices), consequence (experiencing self-care practices) and being patients with HTN in a social context. This chapter also included the study strengths, limitations and conclusion

\section{Start of the disease}

The start of the HTN disease was identified as a causal condition where the patients experienced several symptoms before the beginning of illness. This was consistent with Balduino et al. [24] findings, where the participants noticed something strange with their body before being diagnosed with HTN. Once patients diagnosed with HTN they start to think about self-care. After being diagnosed with HTN, patients start to adjust their self-perception and start to reshape their identity in a different way [24].

\section{Beliefs toward HTN}

In our study, beliefs regarding HTN were one of the identified strategies. Hypertension was perceived as a common, non-threatening disease that can be 
managed by using medications. This congruent with the previous studies; where the participant perceived HTN as a common and non-worrisome problem [34] [35]. In our study, stress, salty and fatty foods were believed to be factors that lead to HTN. Our finding was congruent with one qualitative study aimed to understand Indian immigrant' perception regarding HTN, where the participants identified eating habits, diet rich in salt and fat, tension and stress as cusses for HTN [35].

\section{Beliefs toward HTN self-care practices}

Belief toward HTN self-care practices was another identified strategy. In our study, participants have negative feelings regarding antihypertensive medications; they viewed alternatives medicine as preferred and safe choice. Our findings were consistent with one qualitative study that aimed to identify factors related to noncompliance with antihypertensive medications [34]. In their study, Gascóna et al. [34] found that participants were afraid from medications; they perceived medication as being damaging and not good for body and expressed their desire in finding alternative. Our findings showed that participants have a varying belief regarding the benefit of physical exercise in controlling HTN.

Knowledge and awareness regarding HTN disease and self-care practice

Participants' awareness and knowledge regarding HTN disease and self-care practices were also identified as a strategy. In our study, participants gain their knowledge regarding HTN disease and self-care practices mainly from untrusted resources such as internet, people talks, TV, spice dealers, relatives and not from health care providers. Untrusted resources for HTN information and the lack of participants and health care providers' interaction lead to participants' hesitation regarding the benefit of self-care practices. This in turn might lead to the poor compliance with some aspect of self-care. Our findings were consistent with previous studies, where patients with HTN gain their knowledge from untrusted resources like internet, TV and not from health care providers and this resulted in poor compliance with self-care practices [34] [36]

The participants in our study were aware about the complications of uncontrolled HTN. Participants were also afraid from complications and this motivated them to take care for themselves. A study by Kusuma [35] demonstrated that contrary to our results; participants were not aware for the consequences of uncontrolled BP. In the previous study poor knowledge and lack of awareness were evident among participants [35].

\section{Experiencing self-care practices}

Once the participants identify strategies for the self-care they start to demonstrate varying level of compliance with self-care that are needed to improve their health. This finding is consistent with Balduino et al. [24] study, where the participants understanding for the disease process and their awareness regarding appropriate self-management preceded the development of participants' attitude and actions needed to self-management.

In our study, participants seem to follow HTN diet where they avoided salty and fatty diet and tried to consume fruits and leafy vegetables. This was consis- 
tent with previous findings, where the participants focused on the dietary aspect in term of low salt/fatty diet and diet rich in vegetables to control BP [35].

Despite of the antihypertensive medication proven harm, our participants complied with their medications but it seemed that they were obligated with this compliance. A study conducted by Lubaki et al. [36] demonstrated contrary findings, where the participants reported non-compliance with antihypertensive medication. Noncompliance with medication was related to the participants' uncomfort with medication side effects and to their poor knowledge regarding medication use [26] [36]. One of the reasons for the participants' compliance with antihypertensive medication was due to their awareness with uncontrolled BP complications. Congruent with our findings, a mixed method study aimed to understand factors influenced adherence with antihypertensive medication find that participants' awareness regarding increased BP complications was a factor that enhanced antihypertensive medication compliance [26].

Participants viewed physical activity in different ways and they expressed no specific techniques to control their stress; they depend on God to manage their problems. In one qualitative study, participants emphasized the importance of physical exercise in term of walking and yoga [35]. In the same study, participants viewed stress to be unavoidable and they try to control their anger and be calm without using any techniques.

In relation to the $\mathrm{BP}$ monitoring our participants demonstrated varying practices; where some comply with frequent BP monitoring other did not. In one qualitative study, lack of support was reported to be a factor that prevents participants' to frequently monitor their BP [36].

In the current study participants preferred the alternative medicine and they used natural remedies and herbals to control their BP. Congruent with our findings, one qualitative study aimed to identify factors related to noncompliance with hypertension treatment [34]. The participants in the previous study found alternative medicine to be efficient in controlling their BP.

\section{Being a patient with HTN in a social context}

In our study we discovered that the process of compliance with self-care was impacted by the social and the health care context. The social context has been imposed as a dominant source for information from which participants gain their knowledge regarding self-care practices. On the other hand, the medical context was limited to the disease diagnosis and for reissuing medications. Participants were not provided by any health education by health care providers and no follow up visit was arranged for them. The absence of health care context from the process of self-care has been translated into poor compliance with certain aspects of self-care. These findings were consistent with Lubaki et al. [36] study, where the clinics and follow up visits were mainly for the purpose of diagnosis and no health education were provided to the participants. In the previous study, the ineffective patients-health care provider relationship has led to patients' poor compliance with self-care [36]. 
Different strength of personal beliefs which were impacted by the different context has its impact on participants compliance with self-care. It seems that participants' personal belief with self-care practices addressed within health care context was strong. However, participants seem to be hesitant regarding the aspect of self-care addressed within social context.

\section{Strengths}

The current study addressed the process of compliance with self-care practices from participant' perspective and this considered a key strength since literature tends to study the topic without considering the patient point of view. This study was also rigorously conducted; we recorded the interviews, obtained field notes and we constantly compared data to data, codes to data and codes to categories to ensure the credibility. Additionally, we used semi-structured interview that helped interviewee to freely express their compliance with self-care. Moreover, reflection we used in the current study reduced the subjectivity and helped us to clarify our preconceived ideas about the topic.

\section{Weaknesses}

Because of the use of purposive sampling techniques, the findings of our study cannot be generalized to entire population with HTN. However, qualitative approach not concerns the generalizability rather it aims to explore the multiple realities. Also, the small sample size of the study participants was a limitation. The use of the interview technique for data collection has several limitations such as interviewer bias and power asymmetry.

\section{Conclusions}

The process of compliance with self-care has a path of actions and interactions. The process started from the moment of diagnosis where the patients start to think about self-care. Patients start to form their own understanding and belief regarding HTN. They seek information and start to outline practices to manage their BP.

The process of compliance with self-care practices is highly impacted by the social context where the patients gain their knowledge mainly from untrusted resources. The absence of health care context leads to varying level of compliance with self-care among patients with HTN. This indicated the need for more effective patient and health care provider relationship, education and awareness campaign.

\section{Conflicts of Interest}

The authors declare no conflicts of interest regarding the publication of this paper.

\section{References}

[1] Hussein, A.A., Salam, E.A. and Amr, A.E.S.F. (2017) A Theory Guided Nursing Intervention for Management of Hypertension among Adults at Rural Area. Journal of Nursing Education and Practice, 7, 66-78. https://doi.org/10.5430/jnep.v7n1p66 
[2] Orem, D. (1985) Concepts of Practice. 3rd Edition, McGraw-Hill, New York.

[3] Egan, B.M. and Forman, J.P. (2017) Patient Adherence and the Treatment of Hypertension.

[4] Chobanian, A.V., Bakris, G.L., et al. (2003) Seventh Report of the Joint National Committee on Prevention, Detection, Evaluation, and Treatment of High Blood Pressure. Hypertension, 42, 1206-1252. https://doi.org/10.1161/01.HYP.0000107251.49515.c2

[5] Rigsby, B.D. (2011) Hypertension Improvement through Healthy Lifestyle Modifications. The ABNF Journal Official: Journal of the Association of Black Nursing Faculty in Higher Education, 22, 41-43.

[6] Ea, E.E., Colbert, A., Turk, M. and Dickson, V.V. (2018) Self-Care among Filipinos in the United States Who Have Hypertension. Applied Nursing Research, 39, 71-76. https://doi.org/10.1016/j.apnr.2017.11.002

[7] Gohar, F., Greenfield, S.M., Gareth Beevers, D., Lip, G.Y.H. and Jolly, K. (2008) Self-Care and Adherence to Medication: A Survey in the Hypertension Outpatient Clinic. BMC Complementary and Alternative Medicine, 8, 4.

https://doi.org/10.1186/1472-6882-8-4

[8] Olsen, M.H., Angell, S.Y., Asma, S., Boutouyrie, P., Burger, D., Chirinos, J.A., Wang, J.G., et al. (2016) A Call to Action and a Life Course Strategy to Address the Global Burden of Raised Blood Pressure on Current and Future Generations: The Lancet Commission on Hypertension. The Lancet, 388, 2665-2712.

https://doi.org/10.1016/S0140-6736(16)31134-5

[9] Forouzanfar, M.H., Liu, P., Roth, G.A., Ng, M., Biryukov, S., Marczak, L., Ali, R., et al. (2017) Global Burden of Hypertension and Systolic Blood Pressure of at Least 110 to $115 \mathrm{~mm} \mathrm{Hg}, 1990-2015$. JAMA, 317, 165-182. https://doi.org/10.1001/jama.2016.19043

[10] World Health Organization (2015) Cause Specific Mortality. http://www.who.int/healthinfo/global_burden_disease

[11] Tailakh, A., Evangelista, L.S., Mentes, J.C., Pike, N.A., Phillips, L.R. and Morisky, D.E. (2014) Hypertension Prevalence, Awareness, and Control in Arab Countries: A Systematic Review. Nursing \& Health Sciences, 16, 126-130. https://doi.org/10.1111/nhs.12060

[12] Centers for Disease Controls and Prevention (2014) Effects of High Blood Pressure. https://www.cdc.gov/bloodpressure/effects.htm

[13] Egan, B.M. and Forman, J.P. (2018) The Prevalence and Control of Hypertension in Adults.

[14] Bulatova, N.R., Yousef, A.M., AbuRuz, S.D. and Farha, R.A. (2013) Hypertension Management and Factors Associated with Blood Pressure Control in Jordanian Patients Attending Cardiology Clinic. Tropical Journal of Pharmaceutical Research, 12, 827-833. https://doi.org/10.4314/tjpr.v12i5.25

[15] Jaddou, H.Y., Batieha, A.M., Khader, Y.S., Kanaan, A.H., El-Khateeb, M.S. and Ajlouni, K.M. (2011) Hypertension Prevalence, Awareness, Treatment and Control, and Associated Factors: Results from a National Survey, Jordan. International Journal of Hypertension, 2011, Article ID: 828797.

https://doi.org/10.4061/2011/828797

[16] Khader, A., Farajallah, L., Shahin, Y., Hababeh, M., Abu-Zayed, I., Zachariah, R., Seita, A., et al. (2014) Hypertension and Treatment Outcomes in Palestine Refugees in United Nations Relief and Works Agency Primary Health Care Clinics in Jordan. Tropical Medicine \& International Health, 19, 1276-1283. 
https://doi.org/10.1111/tmi.12356

[17] Horsa, B.A., Tadesse, Y. and Engidawork, E. (2019) Assessment of Hypertension Control and Factors Associated with the Control among Hypertensive Patients Attending at Zewditu Memorial Hospital: A Cross Sectional Study. BMC Research Notes, 12, 152. https://doi.org/10.1186/s13104-019-4173-8

[18] Ostchega, Y., Zhang, G., Hughes, J.P. and Nwankwo, T. (2018) Factors Associated with Hypertension Control in US Adults Using 2017 ACC/AHA Guidelines: National Health and Nutrition Examination Survey 1999-2016. American Journal of Hypertension, 31, 886-894. https://doi.org/10.1093/ajh/hpy047

[19] Bryant, A. and Charmaz, K. (2007) Handbook of Grounded Theory. Sage Publications, London. https://doi.org/10.4135/9781848607941

[20] Charmaz, K. (2006) Constructing Grounded Theory: A Practical Guide through Qualitative Analysis. Sage Publications, Thousand Oaks.

[21] Charmaz, K. (2000) Grounded Theory: Objectivist and Constructivist Methods. 2nd Edition, Sage Publications, London.

[22] Charmaz, K. (2017) Special Invited Paper: Continuities, Contradictions, and Critical Inquiry in Grounded Theory. International Journal of Qualitative Methods, 16, 1609406917719350. https://doi.org/10.1177/1609406917719350

[23] Streubert, H.J. and Carpenter, D.R. (2011) Qualitative Research in Nursing: Advancing the Humanistic Imperative. 5th Edition, Lippincott Williams \& Wilkins, Philadelphia.

[24] Balduino, A.D.F.A., Mantovani, M.D.F., Lacerda, M.R., Marin, M.J.S. and Wal, M.L. (2016) Experience of Hypertensive Patients with Self-Management of Health Care. Journal of Advanced Nursing, 72, 2684-2694. https://doi.org/10.1111/jan.13022

[25] Fix, G.M., Cohn, E.S., Solomon, J.L., Cortés, D.E., Mueller, N., Kressin, N.R., Bokhour, B.G., et al. (2014) The Role of Comorbidities in Patients' Hypertension Self-Management. Chronic Illness, 10, 81-92. https://doi.org/10.1177/1742395313496591

[26] Schuiling-Veninga, C.C., Nguyen, T.B.Y., Vu, T.H., Wright, E.P. and Postma, M.J. (2017) Adherence to Hypertension Medication: Quantitative and Qualitative Investigations in a Rural Northern Vietnamese Community. PLoS ONE, 12, e0171203. https://doi.org/10.1371/journal.pone.0171203

[27] Morton, K., Dennison, L., Bradbury, K., Band, R.J., May, C., Raftery, J., Yardley, L., et al. (2018) Qualitative Process Study to Explore the Perceived Burdens and Benefits of a Digital Intervention for Self-Managing High Blood Pressure in Primary Care in the UK. BMJ Open, 8, e020843.

[28] Rieger, K.L. (2019) Discriminating among Grounded Theory Approaches. Nursing Inquiry, 26, e12261. https://doi.org/10.1111/nin.12261

[29] Whelton, P.K., Carey, R.M., Aronow, W.S., Casey, D.E., Collins, K.J., Himmelfarb, C.D., MacLaughlin, E.J., et al. (2018) 2017

ACC/AHA/AAPA/ABC/ACPM/AGS/APhA/ASH/ASPC/NMA/PCNA Guideline for the Prevention, Detection, Evaluation, and Management of High Blood Pressure in Adults: A Report of the American College of Cardiology/American Heart Association Task Force on Clinical Practice Guidelines. Journal of the American College of Cardiology, 71, e127-e248. https://doi.org/10.1016/j.jacc.2017.11.006

[30] Holloway, I. (2008) AZ of Qualitative Research in Nursing and Healthcare. Wiley-Blackwell, Hoboken.

[31] Barrett, D. and Twycross, A. (2018) Data Collection in Qualitative Research. https://doi.org/10.1136/eb-2018-102939 
[32] Guba, E.G. (1981) Criteria for Assessing the Trustworthiness of Naturalistic Inquiries. ECTJ, 29, 75.

[33] Shenton, A.K. (2004) Strategies for Ensuring Trustworthiness in Qualitative Research Projects. Education for Information, 22, 63-75.

https://doi.org/10.3233/EFI-2004-22201

[34] Gascóna, J.J., Sánchez-Ortuño, M., Llorc, B., Skidmored, D. and Saturnoa, P.J. (2003) Why Hypertensive Patients Do Not Comply with the Treatment: Results from a Qualitative Study. Family Practice, 21, 125-130. https://doi.org/10.1093/fampra/cmh202

[35] Kusuma, Y.S. (2009) Perceptions on Hypertension among Migrants in Delhi, India: A Qualitative Study. BMC Public Health, 9, Article No. 267. https://doi.org/10.1186/1471-2458-9-267

[36] Lubaki, J.P.F., Mabuza, L., Malete, N., Maduna, P. and Ndimande, J.V. (2009) Reasons for Non-Compliance among Patients with Hypertension at Vanga Hospital, Bandundu Province, Democratic Republic of Congo: A Qualitative Study. African Journal of Primary Health Care \& Family Medicine, 1, 107-111. https://doi.org/10.4102/phcfm.v1i1.68 